\title{
Prediction of engineering properties of recycled aged rubberized mixes using GPC
}

\author{
S.-J. Lee ${ }^{1}$, H. Kim ${ }^{2}$, S. N. Amirkhanian ${ }^{2}$ \& K. W. Kim ${ }^{3}$ \\ ${ }^{I}$ Department of Technology, Texas State University, San Marcos, USA \\ ${ }^{2}$ Department of Civil Engineering, Clemson University, USA \\ ${ }^{3}$ Department of Infrastructures Engineering, \\ Kangwon National University, South Korea
}

\begin{abstract}
The recycling of aged rubberized mixes has been an area of interest since crumb rubber modifier (CRM) was first used to modify asphalt binders in hot mix asphalt pavements over 40 years ago. This paper presents the relationship between molecular sizes of recycled aged CRM binders and the engineering properties of recycled aged CRM mixtures. Gel permeation chromatography was utilized to characterize the molecular size change of CRM binders depending on three aging levels and four reclaimed asphalt concrete (RAP) percentages. CRM mixtures were artificially long-term aged in the laboratory, and the aged CRM mixtures were recycled at $0,15,25$, and $35 \%$ using typical recycling guidelines. Specimens of recycled aged CRM mixtures were tested for indirect tensile strength (ITS) in dry and wet conditions, rutting resistance, resilient modulus, and ITS after long-term aging in the laboratory. The results indicated that the compositional changes of recycled aged CRM binders have good correlations with the engineering properties of recycled aged CRM mixtures. Also, the empirical prediction models were developed, and the predicted values showed a high correlation with the properties of recycled aged CRM mixtures.
\end{abstract}

Keywords: recycling, CRM, GPC, engineering properties.

\section{Introduction}

Rubber modified asphalt pavements have become popular because of their advantages, including increased pavement life, decreased traffic noise, reduced maintenance cost, decreased pollution and increased environmental quality 
[1-3]. Meanwhile, the recycling of aged rubberized mixes has been an area of interest since crumb rubber modifier (CRM) was first used to improve asphalt binder properties in asphalt pavements over 40 years ago in the United States. However, there have been limited studies on the subject of the rubberized pavement recycling issue, mostly carried out by several state departments of transportation (DOTs) [4-6].

In general, gel permeation chromatography (GPC) is used as a research tool for identifying molecules in a substance. Primarily, GPC separates asphalt binders into several portions according to their molecular sizes (i.e., LMS, large molecular size; MMS, medium molecular size and SMS, small molecular size). A number of studies have used GPC technique to analyze and characterize asphalt binders.

This research investigated correlation between molecular size of recycled aged CRM binders and engineering properties of the recycled aged CRM mixtures.

\section{Experimental program}

\subsection{Material}

The crumb rubber produced by mechanical shredding at ambient temperature was obtained from one source: -40 mesh $(0.425 \mathrm{~mm})$, which is widely used to produce the field CRM mixtures in South Carolina. Two base binders of PG 5822 and PG 64-22 were used to produce CRM binders throughout the study. The aggregates used for this study were obtained from two different locations and designated as aggregate sources B and L. Hydrated lime, used as an anti-strip additive, was added at a rate of $1 \%$ by dry mass of aggregate.

\subsection{Recycling of aged CRM binders}

The CRM binder was produced in the laboratory by mixing the CRM with the binder at $177{ }^{\circ} \mathrm{C}$ using an open blade mixer at a blending speed of $700 \mathrm{rpm}$ for 30 minutes [2]. The percentage of crumb rubber added for the rubberized binder was $10 \%$ by weight of the base binder. This mixing condition matches the field practices used in South Carolina to produce field mixtures. The rubberized binders were then artificially aged through a series of accelerated aging processes. The aging processes were conducted to produce long-term aged CRM binders in the laboratory, which is expected to have similar aging level to RAP binders.

Virgin CRM binders produced using PG 64-22 binders were used for the mixing of $0 \%$ and $15 \%$ long-term aged (LTA) binders. The virgin binder grade was selected based on the previous research which concluded that in the case of $15 \%$ RAP, the PG grade of the asphalt binder added for the recycling can be the same as that used in $0 \%$ RAP [7]. In case of the $25 \%$ and $35 \%$ LTA binders, the PG 58-22 base binders, one grade softer binder grade, were utilized to produce virgin CRM binders for the mixing [8]. The recycled aged CRM binders were then artificially aged using the RTFO and PAV processes. 


\subsection{Recycling of aged CRM mixes}

CRM mixes were produced through the Superpave mix design procedures, and these mixes were artificially aged using an accelerated aging process (an oven aging for 2 days at $100{ }^{\circ} \mathrm{C}$ ) in the laboratory [9].

The CRM binder produced using a base binder of PG 64-22 was used for $0 \%$ and $15 \%$ mixture recycling. With respect to the $25 \%$ and $35 \%$ recycling, based on the previous research, the base binder of PG 58-22 was selected to produce the virgin CRM binder [8]. The eight CRM mixtures (2 aggregate sources $\times 4$ recycling percentages of $0 \%, 15 \%, 25 \%$, and $35 \%$ ) were designed according to AASHTO M 323-04. Optimum asphalt contents (OAC) were determined from these designs and used to produce the recycled aged CRM mixtures.

\subsection{GPC test}

Waters GPC equipment with computerized software was used for the chromatographic analysis of binders. A differential refractive index meter (Waters 410) was used as a detector. A series of two columns (Waters HR 4E and HR 3) were used for separating constituents of asphalt binder by molecular size. For testing the samples at a constant temperature, the columns were kept at $35{ }^{\circ} \mathrm{C}$ throughout the test in a column oven. The mobile phase was THF flowing at a rate of $1 \mathrm{ml} / \mathrm{min}$.

Each GPC sample dissolved into THF was filtered through a $0.45 \mu \mathrm{m}$ syringe filter prior to injection into the injection module. A $50 \mu$ l of dissolved sample was injected into GPC injector for each test.

The asphalt binder constituents are generally classified into several groups such as asphaltenes, resins, and oils. In this study, a chromatogram profile was partitioned into 13 slices and three parts: large molecular size (LMS; slices 1 to 5), medium molecular size (MMS; 6 to 9) and small molecular size (SMS; 10 to 13). Only the front part, the LMS value, in the quantitative data of the chromatogram was used to characterize the binder properties. Previous research has shown that the LMS of binder had better correlations with asphalt binder properties than other sizes $[10,11]$.

\subsection{Engineering tests}

Indirect tensile strength (ITS) properties were measured to evaluate the moisture susceptibility of the mixtures. Two sets of 3 samples each were tested at $25^{\circ} \mathrm{C}$ in dry and wet states.

Asphalt pavement analyzer (APA) test was conducted to measure rutting resistance on cylindrical samples with an air void content of $4 \pm 0.5 \%$, a height of $75 \mathrm{~mm}$ and a diameter of $150 \mathrm{~mm}$.

Resilient modulus test was carried out at temperatures of 5,25 , and $40{ }^{\circ} \mathrm{C}$ according to ASTM 4123. Four duplicate samples with $150 \mathrm{~mm}$ diameter and 95 $\mathrm{mm}$ thickness were compacted using the Surperpave Gyratory Compactor (SGC) to an air void content of $4 \pm 0.5 \%$. 
Recycled aged CRM mixtures were artificially long-term aged using an accelerated aging process in the laboratory. The ITS tests were conducted to evaluate the properties of recycled aged CRM mixtures after artificial long-term aging. One set of 3 samples for each mixture was tested at $25^{\circ} \mathrm{C}$ in dry state.

\section{Results and discussion}

\subsection{Correlation analysis}

Figures 1 to 3 illustrate the relationship between the LMS values of recycled aged CRM binders and the engineering properties of recycled aged rubberized

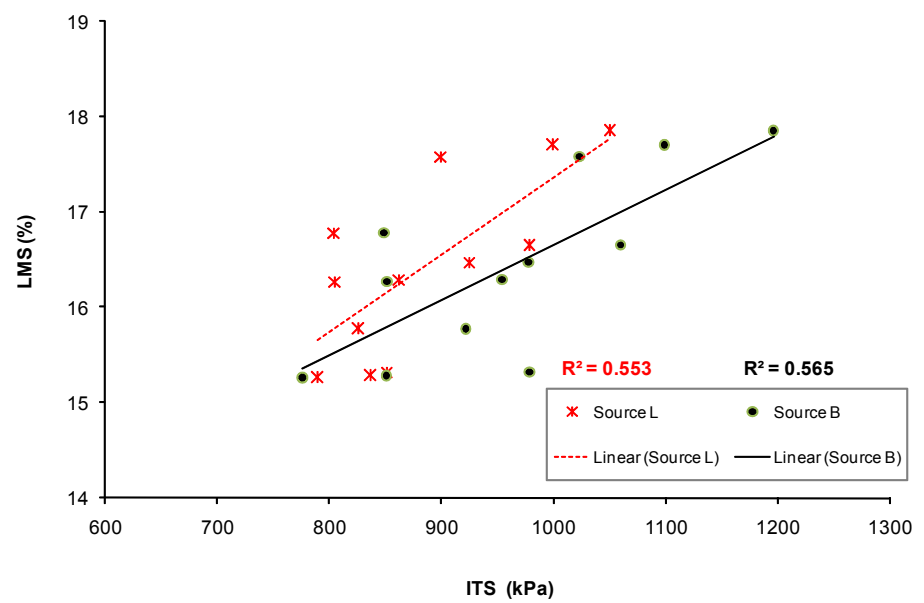

(a)

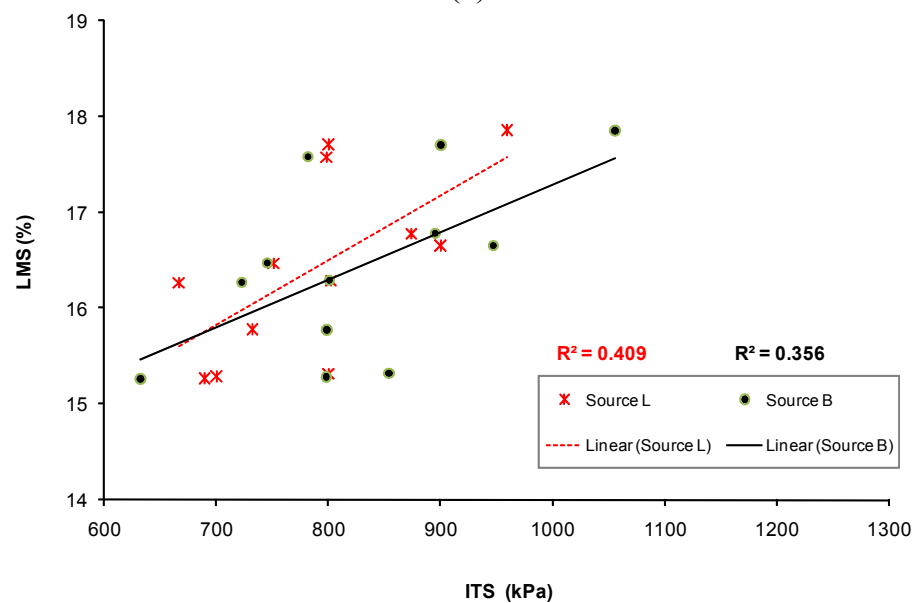

(b)

Figure 1: $\quad$ Relation of the LMS (\%) of recycled aged CRM binders with ITS of recycled aged CRM mixtures: (a) dry ITS and (b) wet ITS. 


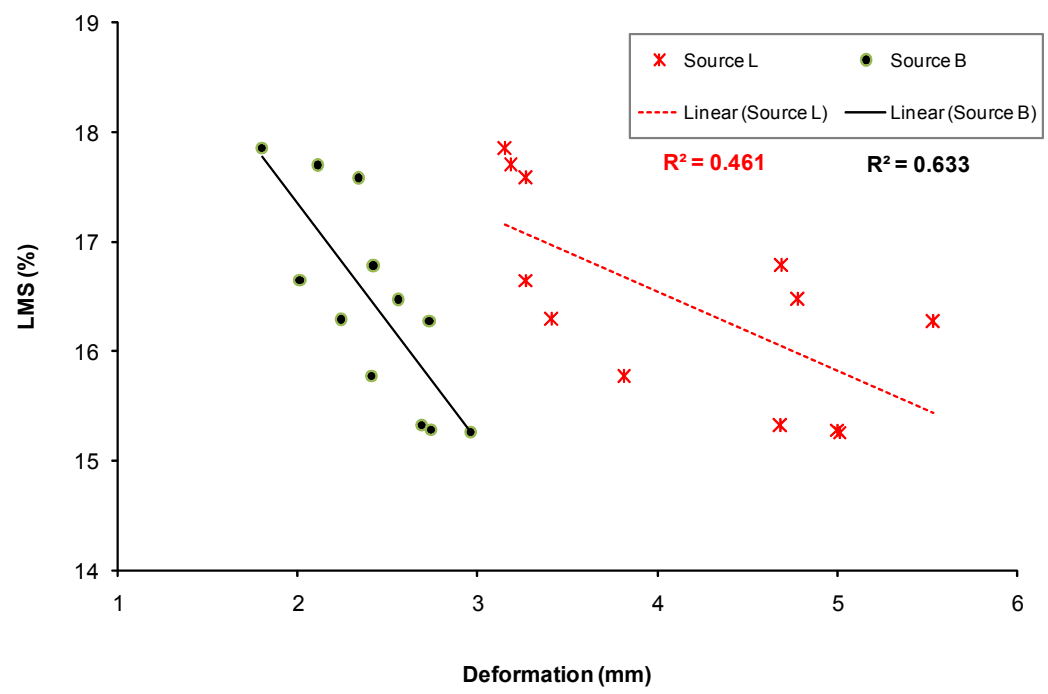

Figure 2: $\quad$ Relation of the LMS (\%) of recycled aged CRM binders with final rut depth of recycled aged CRM mixtures.

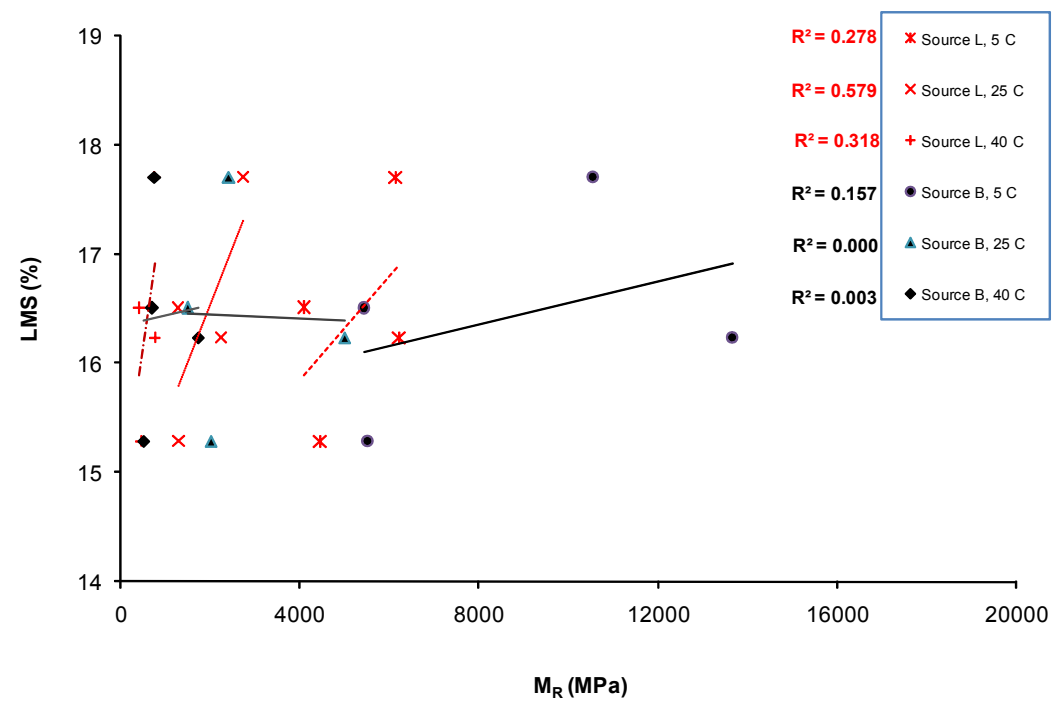

Figure 3: Relation of the LMS (\%) of recycled aged CRM binders with resilient modulus of recycled aged CRM mixtures.

mixtures. It was found that the LMS values were correlated with the dry ITS values of recycled aged CRM mixtures with reasonable $\mathrm{R}^{2}$ values (over 0.55 on the average). The wet ITS values showed somewhat lower correlations (below 
0.39 on the average) with corresponding LMS values than the dry ITS values. This is considered to be associated to the higher air void contents (i.e., $6 \% \sim 8 \%$ ) of samples for ITS test; therefore, increasing the variance of test results, especially in the wet ITS tests.

The deformation values from the APA test generally increased as the LMS values decreased (Figure 2). It was noticed that the higher LMS values resulted in an increase in the rutting resistance of recycled aged CRM mixtures. When compared between each aggregate source, the final rut depth values of the mixtures manufactured with source B resulted in good correlation with the LMS values $\left(R^{2}\right.$ value of 0.633$)$. The rut depth values of mixtures made with source $B$ were relatively lower than those with source L; therefore, indicating that the mixture less susceptible to rutting led to better correlation with the LMS values. Different from the ITS and final rut depth values, it indicated that the resilient modulus values of recycled aged CRM mixtures seemed to have poor correlation with the LMS values (Figure 3). The LMS and resilient modulus properties showed the mean $\mathrm{R}^{2}$ value of 0.223 . A resilient modulus test method is known to have quite high variance of test results, and it is difficult to have consistent trends.

Figure 4 depicts the relation of the LMS values of the CRM binders (RTFO+PAV residual) with dry ITS values of the CRM mixtures after long-term oven aging. As expected, it showed that the higher LMS values caused an increase in the ITS values. The ITS properties of the mixture after long-term aging and LMS values of the binders after RTFO+PAV aging are considered to have excellent relations (the $\mathrm{R}^{2}$ values of 0.829 and 0.808 ), given the variability of long-term aging methods between the CRM binders and the CRM mixtures.

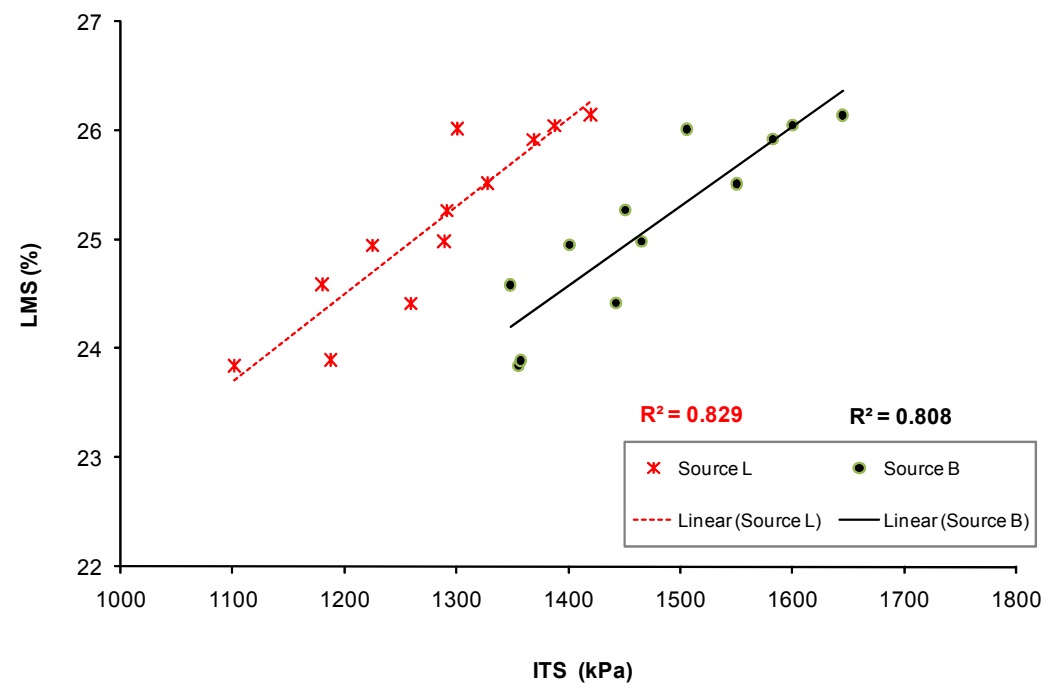

Figure 4: Relation of the LMS (\%) of recycled aged CRM binders (RTFO+PAV residual) with dry ITS of recycled aged CRM mixtures after long-term oven aging. 


\subsection{Regression analysis}

Regression analyses were performed to determine the relationship between the properties of recycled aged CRM mixtures with the LMS values of the binders. Prediction models were developed for two engineering properties of deformation and ITS after long-term oven aging from the LMS values using the STEPWISE procedure of the Statistical Analysis System (SAS) program.

\subsection{Results of regression analysis}

The plots of the measured versus predicted values of the deformation and the ITS (after long-term oven aging) of recycled aged CRM mixtures are presented in Figures 5 and 6 , respectively. It can be observed that the predicted values using the regressions are correlated well with the measured values. Given the variability of properties depending on the aggregate source, the engineering properties of the mixes and the LMS values of the binders are considered to have good relations (the $\mathrm{R}^{2}$ values of 0.753 and 0.748 ).

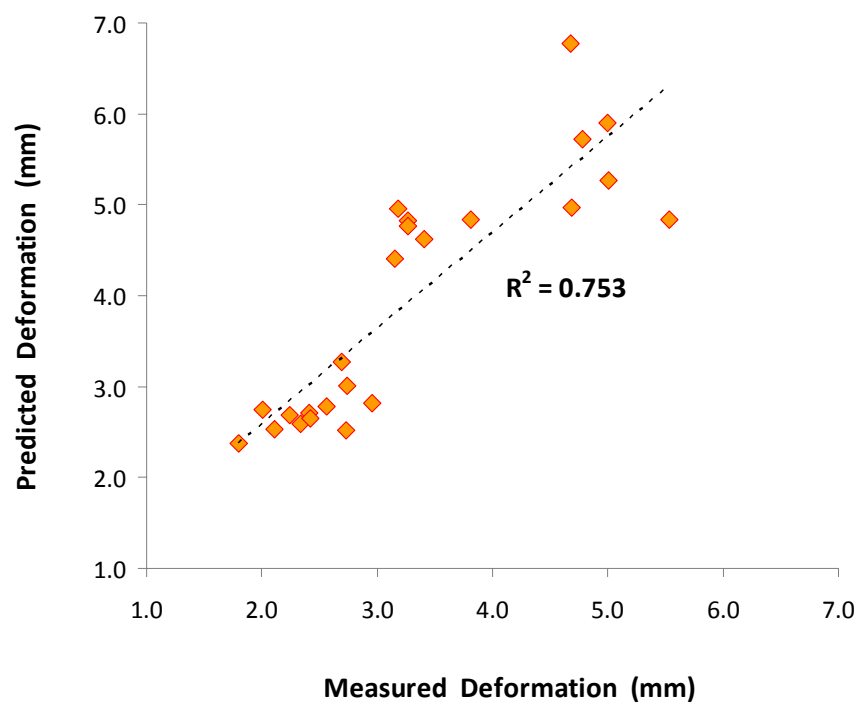

Figure 5: $\quad$ Measured versus predicted deformation values of recycled aged CRM mixes.

\section{Conclusions}

(1) In general, the recycled aged CRM binders with high amount of LMS related to higher tensile strength values of the recycled aged CRM mixtures. Samples in the dry state were found to have higher coefficient of determination $\left(R^{2}\right)$ values than samples in the wet state. 


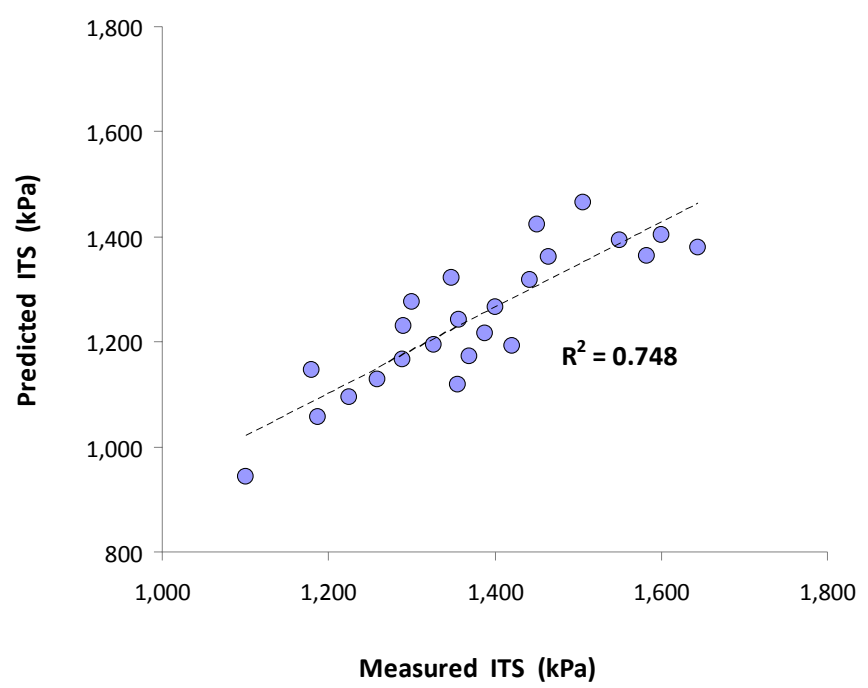

Figure 6: $\quad$ Measured versus predicted dry ITS values of recycled aged CRM mixes after long-term oven aging.

(2) As expected, the higher LMS values of the binders seemed to lead to an increase in the rutting resistance of the mixtures. However, resilient modulus test results were insignificantly correlated with the GPC test results.

(3) Tensile strength properties of recycled aged CRM mixtures after longterm aging were most strongly correlated (the average $R^{2}$ value of 0.819 ) with large molecular size change of recycled aged CRM binders (RTFO+PAV residual).

(4) The empirical models predicting the properties of recycled aged CRM mixes were developed using the GPC parameter of recycled aged CRM binders. The predicted values using the regressions showed a high correlation with the engineering properties of the mixes.

\section{Acknowledgement}

The authors wish to acknowledge and thank South Carolina's Department of Health and Environmental Control (DHEC) for their financial support of this project.

\section{References}

[1] Huang, B., Mohammad, L.N., Graves, P.S., and Abadie, C. (2002). Louisiana Experience with Crumb Rubber-Modified Hot-Mix Asphalt Pavement, Transportation Research Record: Journal of the Transportation Research Board, No. 1789, pp. 1-13. 
[2] Lee, S.-J., Amirkhanian, S., Putman, B., Kim, K. W. A laboratory Study of the Effects of Compaction on the Volumetric and Rutting Properties of CRM Asphalt Mixtures, Journal of Materials in Civil Engineering, ASCE, Vol. 19, No. 12, pp. 1079-1089.

[3] Ruth, B.E. and Roque, R. (1995). Crumb Rubber Modifier (CRM) in Asphalt Pavements, Proceedings of the Transportation Congress, pp. 768 785.

[4] Albritton, G.E., Barstis, W.F. and Gatlin, G.R. (1999). Construction and Testing of Crumb Rubber Modified Hot Mix Asphalt Pavement. Report No: FHWA/MS-DOT-RD-99-115. Federal Highway Administration, Washington D.C.

[5] Bischoff, D. and Toepel, A. (2004). Tire Rubber in Hot Mix Asphalt Pavement. Wisconsin Department of Transportation, Madison, WI.

[6] Crockford, W.W., Makunike, D., Davison, R.R., Scullion, T. and Billiter, T.C. (1995). Recycling Crumb Rubber Modified Pavements. Texas Transportation Institute Research Report 1333-1F. Texas A\&M University, College Station, TX.

[7] Pavement Recycling Guidelines for State and Local Governments (1997). FHWA-SA-98-042.

[8] McDaniel, R., Soleymani, H., Anderson, R.M., Tuner, P. and Peterson, R. (2000). Recommended Use f Reclaimed Asphalt Pavement in the Superpve Mix Design Method, National Cooperative Highway Research Program, Washington, D.C.

[9] Kliewer, J. E., Bell, C. A., and Sosnovske, D. A. (1995). "Investigation of the Relationship between Field Performance and Laboratory Aging Properties of Asphalt Mixtures." ASTM STP 1265, pp. 3-20.

[10] Kim, K. W., Doh, Y. S. and Amirkhanian, S. (2004). Effect of Polymers on Aging of Asphalt Binder, Journal of Korean Society of Pavement Engineers, Vol. 6, No. 2, pp. 15-24.

[11] Jennings, P. W. (1980). High Pressure Liquid Chromatography as a Method of measuring Asphalt Composition, Publication FHWA-MT-7930, FHWA, U. S. Department of Transportation. 